\title{
Clinicopathological features of idiopathic membranous nephropathy combined with IgA nephropathy: a retrospective analysis of 9 cases
}

\author{
Ruimin $\mathrm{Hu}^{1,2}$, Guolan Xing ${ }^{2^{*}}$, Huijuan $\mathrm{Wu}^{1}$ and Zhigang Zhang ${ }^{1^{*}}$ (D)
}

\begin{abstract}
Background: The concomitant presence of idiopathic membranous nephropathy and IgA nephropathy is rare. Here, we report 9 cases of phospholipase-A2-receptor (PLA2R) positive idiopathic membranous nephritis combined with IgA nephropathy, while reviewing publications regarding the pathological characteristics of this glomerolonephritis complication.

Case presentation: Nine cases of renal biopsy tissues were retrospectively reviewed, including the clinicopathological features, the results of the immunofluorescence assays, and the electron microscopic examination. The patients mainly presented proteinuria and microscopic hematuria, and the serum anti-PLA2R was detected as positive in all of the patients. Histologically, a wide thickening of the glomerular basement membrane was observed in each of the 9 cases. Additionally, there existed mild hyperplasia in the mesangial cell and the matrix of the mesangial area. Immunofluorescence assays showed prominent glomerular granular staining on the glomerular capillary loops for lgG $(++/+++)$, IgG4 (++/++++), and PLA2R (+/++). In addition, moderate IgA positive stains were focally or sparsely limited to the mesangial areas. Electron microscopy revealed subepithelial and mesangial electron-dense deposits.

Conclusions: The results from the case analyses indicated that idiopathic membranous nephropathy combined with IgA nephropathy possess the clinicopathological features found in both components. It is suggested that serum anti-PLA2R and tissue PLA2R are important biomarkers that can assist in the diagnosis of idiopathic membranous nephropathy associated with IgA nephropathy.
\end{abstract}

Keywords: Idiopathic membranous nephropathy, IgA nephropathy, PLA2R

\section{Background}

Membranous nephropathy (MN) and IgA nephropathy (IgAN) are two distinct glomerular diseases. The most common cause of nephrotic syndrome in adults can be attributed to $\mathrm{MN}$, in which approximately $70 \%$ of this disease can be categorized as idiopathic MN (also referred to as primary MN). In Chinese as well as other

\footnotetext{
* Correspondence: xg|@zzu.edu.cn; zzg@shmu.edu.cn

${ }^{2}$ Department of Nephrology, The First Affiliated Hospital of Zhengzhou University, 1, Jianshe Road East, Erqi District, Zhengzhou, Henan 450052, People's Republic of China

'Department of Pathology, School of Basic Medical Sciences, Fudan University, 138, Yixueyuan Road, Xuhui District, Shanghai 200032, People's Republic of China
}

(c) 2016 The Author(s). Open Access This article is distributed under the terms of the Creative Commons Attribution 4.0 International License (http://creativecommons.org/licenses/by/4.0/), which permits unrestricted use, distribution, and reproduction in any medium, provided you give appropriate credit to the original author(s) and the source, provide a link to the Creative Commons license, and indicate if changes were made. The Creative Commons Public Domain Dedication waiver (http://creativecommons.org/publicdomain/zero/1.0/) applies to the data made available in this article, unless otherwise stated. logical type of glomerular disease. Furthermore, it was estimated that IgAN accounts for 45.2 to $58.2 \%$ of primary glomerular diseases in China [1-3]. Recently, an increasing number of reports have emerged in regards to IgAN combined with other renal diseases $[1,4]$. However, studies involving the concomitant presence of idiopathic $\mathrm{MN}$ and IgAN are rare. The few reports available from studies conducted around the world rely heavily on immunofluorescence and electron microscopic examination to identify patients with combined idiopathic MN and IgAN. These data showed subepithelial and mesangial electron-dense 
deposition (EDD) in which the immunofluorescence analysis conducted on the patient samples was positive for both IgG and IgA [5-10]. However, it may bring some confusion in diagnosing these complications by immunofluorescence and electron microscopic examination, because some secondary $\mathrm{MN}$ also showed mesangial EDD in additional to subepithelial deposition, while the IgAN sometime have minimal subepithelial EDD with mesangial massive deposition.

Recently, serum anti-phospholipase-A2-receptor (PLA2R) antibodies were found to be the main serum autoantibodies presented in idiopathic MN patients. Furthermore, previous reports have demonstrated that M-type PLA2R is a specific marker for idiopathic MN $[11,12]$. It has been shown that PLA2R is hardly detected in secondary $\mathrm{MN}$ and other glomerular diseases $[13,14]$. Therefore, the test of anti-PLA2R as a sensitive biomarker may provide a novel diagnostic tool for idiopathic $\mathrm{MN}$, and for the differentiation of secondary $\mathrm{MN}[11,15,16]$. This biomarker also provides a more definitive evidence for detecting idiopathic MN combined with IgAN in patients. In this article, we report 9 cases of PLA2R-positive idiopathic MN combined with IgAN, in addition to a review that discusses and compares our findings with previous publications.

\section{Materials and methods \\ Patients}

Between January 2013 and December 2015, 9 cases of patients with MN combined with IgAN were selected from the nephrosis laboratory of the Department of Pathology, School of Basic Medical Sciences, Fudan University, Shanghai, China and The First Affiliated Hospital of Zhengzhou University, Zhengzhou, China.

\section{Data collection}

These cases are comprised of patients whose renal biopsies showed glomerular predominant IgG and PLA2R deposits with more or less mesangial IgA deposition, excluding the cases of differentiating renal diseases such as lupus nephritis, HBV associated nephritis and antineutrophil cytoplasmic autoantibody (ANCA)-associated crescentic glomerular nephritis (GN) with mesangial IgA deposits. The medical records of the patients were reviewed for age, gender, type and degree of hematuria, urinary protein excretion $(\mathrm{g} / 24 \mathrm{~h})$, and serum creatinine at the time of biopsy.

\section{Biopsy evaluation}

Histological slides from each biopsy were stained with hematoxylin-eosin, periodic acid-Schiff, silver methenamine, and masson's trichrome. In addition, immunofluorescent staining of immunoglobulins, complement, and PLA2R were conducted. These stained samples as well as all of the transmission electron micrographs taken at the time of initial biopsy evaluation were reviewed by two renal pathologists (Dr. Wu and Dr. Zhang).

\section{Case presentation}

The clinical and serologic features of the 9 patients, 4 females and 5males, were summarized in Table 1 . The mean age was $38.3 \pm 7.4$ years (range $25-48$ years). The patients had no history of renal disease and bacterial infection prior to the occurrence of renal complications. The patients had nephrotic syndrome and the mean proteinuria level was $4.82 \pm 3.33 \mathrm{~g} / 24 \mathrm{~h}$ (range 1.79$11.76 \mathrm{~g} / 24 \mathrm{~h}) .7$ of the 9 patients had microscopic hematuria, whereas all patients had no gross hematuria. The mean serum creatinine at the time of biopsy was $68.67 \pm 17.55 \mu \mathrm{mol} / \mathrm{L}$ (range $49-93 \mu \mathrm{mol} / \mathrm{L}$ ). All patients had increased serum PLA2R antibody prominently with a mean level of $57.39 \pm 47.89 \mathrm{RU} / \mathrm{mL}$ (range 24.6$177.1 \mathrm{RU} / \mathrm{mL}$ ), while the serum IgA and complement $\mathrm{C} 3$ were closely distributed within the normal range in these 9 patients. 8 of the 9 cases had no hypertension history, and only case 2 had hypertension for more than 10 years (92-100/136-145 mmHg controlled by extended release nifedipine tablets). The ANA,ANCA and HBV markers detected at the time of biopsy were negative for each of the 9 cases.

Table 2 lists the summary of the histopathological features of the renal biopsies conducted within each of the 9 cases. The biopsies showed a predominantly diffuse thickening of the basement membrane of glomerular capillary walls. Mildly mesangial hyperplasia was observed within 4-5 mesangial cells as well as a small portion of the matrix in all 9 cases. Moreover, focal glomerular tuft necrosis or crescents were absent, while mild chronic interstitial inflammation was present in the patient samples.

In the immunofluorescence staining, each of the cases showed prominent glomerular granular staining on glomerular capillary loops for IgG $(++/+++)$, IgG4 $(++/+++)$, and PLA2R $(+/++)$; C3 $(+/+++)$ also displayed granular staining on glomerular capillary loops. IgA $(+/++)$ positive staining were focally or sparsely limited to the mesangial areas (Fig. 1a-e).

For the electron microscopy, granular EDD were present in the subepithelium in 9 patients, in addition to irregular thickening of the glomerular basement membrane and segmental spikes. The extensive fusion of epithelial foot processes was also observed. Mild, focal proliferation of mesangial cells and the matrix were noted (Fig. 1f). 
Table 1 Summary of the clinical and serological features of renal biopsies from 9 patients

\begin{tabular}{|c|c|c|c|c|c|c|c|c|c|}
\hline & Ca.1 & Ca.2 & Ca.3 & Ca.4 & Ca.5 & Ca.6 & Ca.7 & Ca.8 & Ca.9 \\
\hline Age & 41 & 42 & 28 & 42 & 36 & 25 & 41 & 48 & 42 \\
\hline Sex & F & F & M & M & M & M & F & $\mathrm{F}$ & M \\
\hline Prodromal infection history & Non & Non & Non & Non & Non & Non & Non & Non & Non \\
\hline Family history of kidney diseases & Non & Non & Non & Non & Non & Non & Non & Non & Non \\
\hline Gross hematuria & Non & Non & Non & Non & Non & Non & Non & Non & Non \\
\hline Hypertension & Non & $>10 y s$ & Non & Non & Non & Non & Non & Non & Non \\
\hline 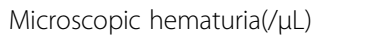 & 37 & 8.58 & 29 & 27 & 15.18 & 45 & 0 & 40 & 0 \\
\hline Proteinuria & +++ & ++ & +++ & +++ & ++++ & +++ & ++ & +++ & +++ \\
\hline 24TP(g/24 h) & 2.97 & 1.79 & 2.28 & 7.61 & 11.76 & 2.59 & 2.4 & 6.42 & 5.52 \\
\hline Serum creatinine $(\mu \mathrm{mol} / \mathrm{L})$ & 49 & 68 & 90 & 64 & 93 & 66 & 52 & 49 & 87 \\
\hline Serum ALB(g/L) & 25.8 & 32.8 & 34.6 & 21.2 & 20.6 & 44.9 & 36.1 & 23.5 & 27.4 \\
\hline Serum $\operatorname{lgA}(g / L)$ & 1.12 & 3.32 & 1.36 & 2.58 & 1.84 & 3.59 & 1.11 & 4.8 & 2.44 \\
\hline Serum C3(g/L) & 0.87 & 1.45 & 0.93 & 1.34 & 1.05 & 1.08 & 0.95 & 1.32 & 1.01 \\
\hline Serum anti-PLA2R(RU/mL) & 69.1 & 34.6 & 31.5 & 53.4 & 177.1 & 27 & 24.6 & 32.9 & 66.3 \\
\hline Serum autoantibodies & - & - & - & - & - & - & - & - & - \\
\hline Serum HBV markers & - & - & - & - & - & - & - & - & - \\
\hline ANCA & - & - & - & - & - & - & - & - & - \\
\hline
\end{tabular}

Note: $C a$. case, $F$ female, $M$ male

Table 2 Summary of the histopathological features of renal biopsies of the 9 patients

\begin{tabular}{|c|c|c|c|c|c|c|c|c|c|}
\hline & Ca.1 & Ca.2 & Ca.3 & Ca.4 & Ca.5 & Ca.6 & Ca.7 & Ca. 8 & Ca.9 \\
\hline \multicolumn{10}{|l|}{ LM } \\
\hline \%globally sclerotic glomeruli & $0 / 30$ & $1 / 23$ & $0 / 18$ & $0 / 33$ & $0 / 24$ & $1 / 18$ & $1 / 38$ & $0 / 20$ & $1 / 31$ \\
\hline \%segmentally sclerotic glomeruli & $0 / 30$ & $0 / 23$ & 0/18 & $0 / 33$ & $0 / 24$ & $0 / 18$ & $1 / 38$ & $0 / 20$ & $1 / 31$ \\
\hline Mesangial cell proliferation & + & + & + & + & + & + & + & - & + \\
\hline Mesangial matrix hyperplasia & + & + & + & + & + & + & + & + & + \\
\hline Thickening of the capillary walls & ++ & + & ++ & ++ & ++ & ++ & ++ & ++ & ++ \\
\hline Interstitial inflammatory & + & + & + & + & \pm & - & + & - & - \\
\hline Focal tubular atrophy & + & + & + & + & - & - & - & - & + \\
\hline \multicolumn{10}{|l|}{ IF } \\
\hline $\lg G$ & +++ & ++ & +++ & +++ & +++ & ++ & ++ & ++ & +++ \\
\hline $\lg A$ & $++(f)$ & + & ++ & $+(f)$ & ++ & ++ & ++ & ++ & ++ \\
\hline $\lg G 1$ & $+/++$ & \pm & ++ & - & ++ & + & - & + & - \\
\hline $\lg G 2$ & \pm & + & - & - & \pm & - & \pm & \pm & + \\
\hline $\lg G 3$ & \pm & $+/++$ & - & + & + & - & \pm & \pm & - \\
\hline $\lg G 4$ & ++ & ++ & ++++ & ++ & +++ & ++ & ++ & ++ & ++ \\
\hline PLA2R & + & + & + & ++ & $+/++$ & + & + & + & + \\
\hline $\mathrm{C} 3$ & $+/++$ & + & ++ & $+/++$ & +++ & + & $+/++$ & + & ++ \\
\hline \multicolumn{10}{|l|}{ EM } \\
\hline Subepithelium EDD & ++ & ++ & ++ & ++ & ++ & ++ & +++ & ++ & ++ \\
\hline Mesangial area EDD & ++ & + & + & + & + & + & ++ & + & + \\
\hline Extensive processes effacement & ++ & ++ & ++ & ++ & $+/++$ & + & ++ & ++ & ++ \\
\hline Mesangial matrix hyperplasia & + & - & + & + & - & - & + & - & + \\
\hline
\end{tabular}




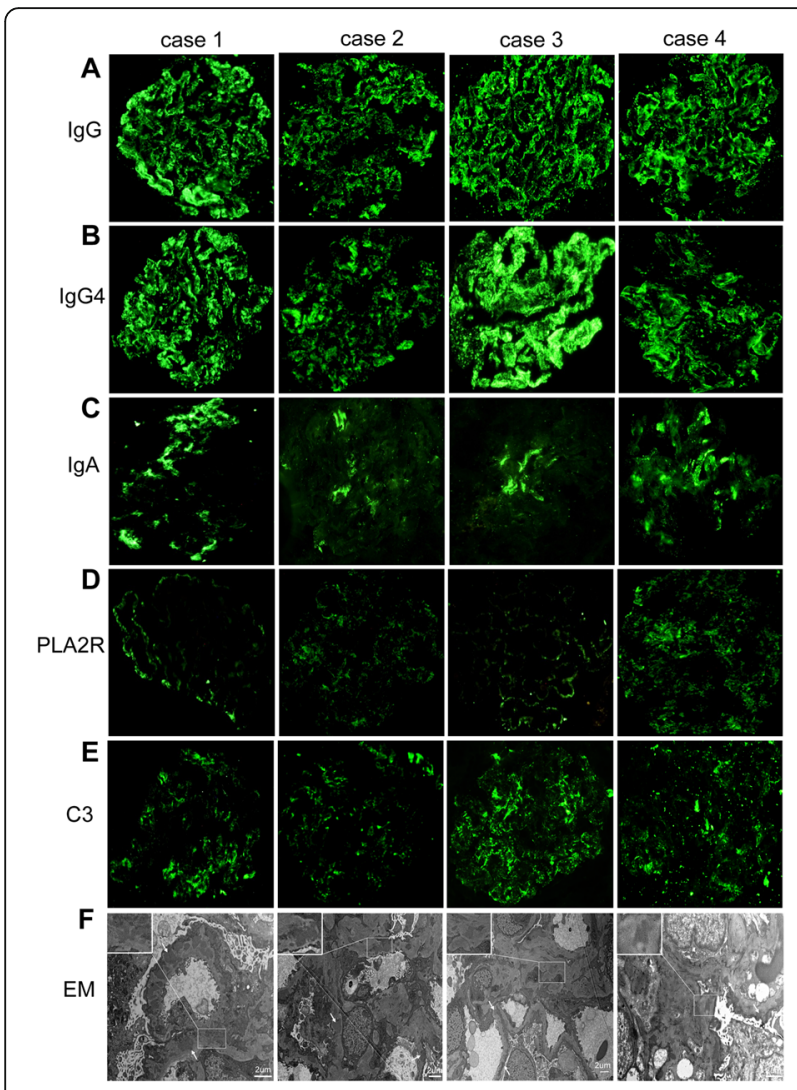

Fig. 1 Cases 1-4 immunofluorescence for immunoglobulins and EDD detected by electron microscopy. Immunofluorescence in cases 1-4 for a lgG, b IgG4, c IgA, d PLA2R and e C3. f The EDD detected by electron microscopy in case $1-4$. White arrows, subepithelial deposits; Inset: boxed mesangial deposits

\section{Discussion and conclusion}

We examined the clinical and morphological features of samples collected from each of the 9 cases of idiopathic MN combined with IgAN. This complication of GN was defined morphologically, with emphasis on the presence of the PLA2R antibody in serum and PLA2R expression in glomeruli, rather than on the basis of the immunofluorescence assay and transmission electron microscopy.

$\mathrm{MN}$ and IgAN are both glomerular diseases mediated by immune complex deposition. The depositing site of the immune complex is different in these two forms of GN due to the diverse mechanisms associated with immune complex formation. These mechanisms are influenced by many factors such as the relative molecular weight and the volume and charge of the immune complex, which may be the cause of the different clinical manifestations and pathological morphology of the two renal diseases. It is found that idiopathic MN with IgAN complications combine the clinical and pathological manifestations of these two types of glomerular diseases. Previous reports regarding patients presenting idiopathic
MN with IgAN show that the average age of the patients was 40 years old. Furthermore, the patients in those studies had proteinuria, of which $50 \%$ exhibited heavy proteinuria with nephrotic syndrome. $90 \%$ of the patients examined in these reports presented microscopic hematuria, and $30 \%$ of these patients presented hypertension [6-10]. In this group, the clinical and pathological features of the 9 cases examined were similar to that found in previous reports. These findings are consistent with the clinical characteristics of MN and IgAN. However, the differences noted in the previous reports and the current study are that the age of renal disease onset in the 9 cases is younger than that in the simple idiopathic MN, and that the deposition of IgA EDD was lesser compared to IgG EDD in patients of $\mathrm{MN}$ combined with IgAN.

In the present study, the immunofluorescence staining showed granular positive IgG along the capillary loop, while IgA was shown to be irregularly positive in the mesangial area. Meanwhile, the EDD of the subepithelial and mesangial area were found using electron microscopy. The mixed immunoglobulins deposition phenomenon is common in many secondary glomerulonephritis, which may cause confusion for diagnosis and differentiation. Wang et al. reported that use of the colloidal gold-immune electron microscopy technique displayed an IgG positive subepithelial deposition, while the anti-IgA was positive in the mesangial deposition, confirming the different location of IgG and IgA in the complication [10].

Idiopathic $\mathrm{MN}$ is recognized as an autoimmune disease [17]. Recently, progress has been made in understanding the pathogens of idiopathic $\mathrm{MN}$ with the finding of M-type PLA2R as the target antigen of podocytes in patients with idiopathic $\mathrm{MN}[11,12]$. It has been revealed that a majority of patients with idiopathic $\mathrm{MN}$ were found to possess anti-PLA2R autoantibodies in circulation, tissue PLA2R expression in their biopsy kidney tissue, and that the level of autoantibody correlates with the level of proteinuria $[11,18]$. Moreover, Beck et al. along with other investigators showed that serum anti-PLA2R or tissue PLA2R is high detect ability in idiopathic $\mathrm{MN}$, but both are rarely detected in secondary MN [15]. Hoxha et al. found that a high ratio of PLA2R positive in the stained glomeruli of patients with idiopathic MN tightly correlates with the presence of serum PLA2R autoantibodies, while this correlation is lower in secondary MN with respect to PLA2R staining [16]. This method of detection may help discriminate between idiopathic $\mathrm{MN}$ from secondary MN. In the 9 cases presented herein, we observed increased serum anti-PLA2R, and glomerular PLA2R positive immunofluorescence staining, associated with IgG subepithelial deposits,and immunofluorescence positive of mesangial 
IgA and mesangial EDD deposition. Therefore, these patients were confirmed as idiopathic $\mathrm{MN}$ combined with IgAN. It is suggested that PLA2R determination is an important assistant biomarker for the diagnosis of idiopathic MN associated with IgAN.

Of note, the 9 cases of idiopathic MN combined with IgAN presented herein had predominant IgG4 and PLA2R co-localized within the subepithelial immune deposits. IgG subtype analysis has been suggested that IgG4-predominant staining is mainly associated with idiopathic MN, whereas IgG1, IgG2, and IgG3 predominate in the deposits of secondary MN [11, 19-23]. Preliminary studies suggest that there may be a direct interaction of anti-PLA2R-IgG4 with the PLA2R molecule, and this interaction activates complement by binding the Fc portion of IgG4 [24]. Furthermore, antiPLA2R-IgG4 has been described as a predominant circulating IgG subclass in idiopathic MN. The presence of these combined molecules seem to be specific ( $89 \%)$ to idiopathic MN $[13,25,26]$ and can be utilized as supportive data to exclude the presence of secondary $\mathrm{MN}$ [13]. Herein, the analysis of the presented cases reveal the significant implications for the diagnosis of idiopathic MN complicated with other renal diseases.

\section{Abbreviations}

ANCA: Anti-neutrophil cytoplasmic autoantibody; EDD: Electron-dense deposition; GN: Glomerular nephritis; IgAN: IgA nephropathy; MN: Membranous nephropathy

\section{Acknowledgments}

We thank Muyi Guo, Yingzi Wang and Songxia Quan for constructive suggestions for the manuscript and Zhonghua Zhao for technical assistance. Permission to acknowledge have been obtained from all these mentioned in the Acknowledgements.

\section{Funding}

None.

\section{Availability of data and materials}

All datasets on which the conclusions of the paper rely upon will be freely available to any scientist for non-commercial purposes upon request, without breaching participant confidentiality.

\section{Authors' contributions}

ZZ, GX and RH collected the clinical information from the 9 cases; ZZ and HW retrospectively reviewed thecases of renal biopsy tissues; $\mathrm{RH}$ and HW drafted the manuscript; ZZ revised and finalized the manuscript. All authors read and approved the final manuscript.

\section{Competing interests}

The authors declare that they have no competing interests.

\section{Consent for publication}

Written informed consent was obtained from each of the patients examined under this study for publication of this case report and any accompanying images. A copy of the written consent from each patient is available for review by the editor of this journal.

\section{Ethics approval and consent to participate}

The study procedures were approved by the hospital ethical committee of the First Affiliated Hospital of Zhengzhou University (reference number: 3).
Received: 19 April 2016 Accepted: 2 September 2016

Published online: 13 September 2016

\section{References}

1. Donadio JV, Grande JP. IgA nephropathy. N Engl J Med. 2002;347:738-48.

2. Li LS, Liu ZH. Epidemiologic data of renal diseases from a single unit in China: analysis based on 13,519 renal biopsies. Kidney Int. 2004;66:920-3.

3. Zhou FD, Zhao MH, Zou WZ, et al. The changing spectrum of primary glomerular diseases within 15 years: a survey of 3331 patients in a single Chinese centre. Nephrol Dial Transplant. 2009;24:870-6.

4. Ge YT, Liao JL, Liang W, et al. Anti-Glomerular Basement Membrane Disease Combined with IgA Nephropathy Complicated with Reversible Posterior Leukoencephalopathy Syndrome: An Unusual Case. Am J Case Rep. 2015;16:849-53.

5. Yoshimura M, Kida $H$, Abe $T$, et al. Significance of IgA deposits on the glomerular capillary walls in IgA nephropathy. Am J Kidney Dis. 1987;9:404-9.

6. Kato A, Kimura M, Fujigaki Y, et al. A case of IgA nephropathy complicated with membranous nephropathy. Nihon Naika Gakkai Zasshi. 1991;80:272-3.

7. Jennette JC, Newman WJ, Diaz-Buxo JA. Overlapping IgA and membranous nephropathy. Am J Clin Pathol. 1987;88:74-8.

8. Doi T, Kanatsu K, Nagai $\mathrm{H}$, et al. An overlapping syndrome of IgA nephropathy and membranous nephropathy? Nephron. 1983;35:24-30.

9. Stokes MB, Alpers CE. Combined membranous nephropathy and IgA nephropathy. Am J Kidney Dis. 1998;32:649-56.

10. Wang SX, Zou WZ, Yang $L$, et al. Clinicopathologic features of membranous nephropathy coexisting with IgA nephropathy. Zhonghua Bing Li Xue Za Zhi. 2007;36:171-4.

11. Beck $L$, Bonegio $R G$, Lambeau $G$, et al. M-type phospholipase $A 2$ receptor as target antigen in idiopathic membranous nephropathy. N Engl J Med. 2009:361:11-21.

12. Debiec $\mathrm{H}$, Guigonis V, Mougenot B, et al. Antenatal membranous glomerulonephritis due to anti-neutral endopeptidase antibodies. N Engl J Med. 2002:346:2053-60.

13. Qin $W$, Beck $L$, Zeng $C$, et al. Anti-phospholipase A2 receptor antibody in membranous nephropathy. J Am Soc Nephrol. 2011;22:1137-43.

14. Gunnarsson I, Schlumberger W, Ronnelid J. Antibodies to M-type phospholipase A2 receptor (PLA2R) and membranous lupus nephritis. Am J Kidney Dis. 2012;59:585-6.

15. Beck LJ, Salant DJ. Membranous nephropathy: from models to man. J Clin Invest. 2014;124:2307-14

16. Hoxha E, Kneissler U, Stege G, et al. Enhanced expression of the M-type phospholipase A2 receptor in glomeruli correlates with serum receptor antibodies in primary membranous nephropathy. Kidney Int. 2012;82:797-804.

17. Makker SP, Tramontano A. Idiopathic membranous nephropathy: an autoimmune disease. Semin Nephrol. 2011;31:333-40.

18. Kanigicherla D, Gummadova J, McKenzie EA, et al. Anti-PLA2R antibodies measured by ELISA predict long-term outcome in a prevalent population of patients with idiopathic membranous nephropathy. Kidney Int. 2013;83:940-8.

19. Donadio JJ, Torres VE, Velosa JA, et al. Idiopathic membranous nephropathy: the natural history of untreated patients. Kidney Int. 1988;33:708-15.

20. Schieppati A, Mosconi L, Perna A, et al. Prognosis of untreated patients with idiopathic membranous nephropathy. N Engl J Med. 1993;329:85-9.

21. Wakai $S$, Magil AB. Focal glomerulosclerosis in idiopathic membranous glomerulonephritis. Kidney Int. 1992;41:428-34.

22. Lee HS, Koh HI. Nature of progressive glomerulosclerosis in human membranous nephropathy. Clin Nephrol. 1993;39:7-16

23. Segal PE, Choi MJ. Recent advances and prognosis in idiopathic membranous nephropathy. Adv Chronic Kidney Dis. 2012;19:114-9.

24. Ma H, Beck LH, Salant DJ. Membranous nephropathy-associated antiphospholipase A2 receptor IgG4 autoantibodies activate the lectin complement pathway [abstract]. J Am Soc Nephrol. 2011;22:62A.

25 Beck LJ, Fervenza FC, Beck DM, et al. Rituximab-induced depletion of anti-PLA2R autoantibodies predicts response in membranous nephropathy. J Am Soc Nephrol. 2011;22:1543-50.

26 Hoxha E, Harendza S, Zahner G, et al. An immunofluorescence test for phospholipase-A(2)-receptor antibodies and its clinical usefulness in patients with membranous glomerulonephritis. Nephrol Dial Transplant. 2011;26:2526-32. 